\title{
Evaluation of Bacterial Biofilm as Biosensor for Detecting Phenol, Catechol, and 1,2-dihydroxynaphthalene
}

\author{
Dyah Iswantini' ${ }^{*}$, Ali Aulia Ghozali ${ }^{2}$, Cecep Kusmana ${ }^{3}$, Novik Nurhidayat ${ }^{4}$ \\ 'Department of Chemistry, Faculty of Mathematics and Natural Sciences, IPB-University, Bogor, Indonesia \\ ${ }^{2}$ Graduated Program of Environmental and Natural Resources Management Sciences, IPB-University, Bogor, Indonesia \\ ${ }^{3}$ Department of Silviculture, Faculty of Forestry, IPB-University, Bogor, Indonesia \\ ${ }^{4}$ Microbial Laboratory for Health, Central Research Department of Biology, Indonesian Institute of Sciences (LIPI), Cibinong, Indonesia
}

\section{ARTICLE INFO}

Article history:

Received February 4, 2021

Received in revised form July 1, 2021

Accepted August 23, 2021

\section{KEYWORDS:}

Bacillus amyloliquifaciens,

biosensor,

electrochemistry Pseudomonas aeruginosa,

Staphylococcus sciuri,

voltammetry cyclic

\begin{abstract}
Monitoring of water quality from the presence of polyaromatic hydrocarbon (PAHs) compounds and its derivates are important for keeping the healthy aquatic environment. Some of those derivates are phenol and several related compounds sharing simmilar structures. This reseach aimed for the detection of those phenol and several similar compounds monitoring due to PAHs degradation. Three identified bacterial isolates of Pseudomonas aeruginosa, Staphylococcus sciuri, and Bacillus amyloliquifaciens were selected based on their phenol degradation characters. On physiological properties all three isolates were observed to degrade several hydrophobic substances such as for naphthalene and anthracene. Yet, genetic analysis indicated that the phenolic degradating oxygenase gene was detected only in the $\boldsymbol{P}$. aeruginosa and $\boldsymbol{S}$. sciuri. Applying those isolates for biofilm as biosensor showed a sufficient analytical performance such as their limit of detection between 0.1-0.5 $\mu \mathrm{M}$.
\end{abstract}

\section{Introduction}

Hydrocarbon aromatic compounds is a term of compound group which shares the same main structure, the aromatic system, as found in BTX (benzene, toluene, and xylene) and PAHs (polycyclic aromatic hydrocarbons). Naturally, aromatic compounds can be formed through incomplete combustion (Pampanin and Sydnes 2012). Aromatic compounds are usually used as the indication of hydrocarbon compound contamination in the environment (Minarghi et al. 2019). Aromatic compounds are able to be degraded through oxidative degradation, where some electrochemically active compounds; like cathecol, phenol, and many other similar compounds sharing similar structure, emerge (Liu et al. 2014). Thus, hypothetically it is feasible to detect them electrochemically.

Conventionally, the monitoring of aromatic compounds are measured using HPLC (high performance liquid chromatography) or GC-MS (gas chromatography-mass spectrometry) (FernandezValera et al. 2010; Yin et al. 2015). Both methods are not practical due to extraction process and high energy requirement. Considering the methabolical

\footnotetext{
* Corresponding Author

E-mail Address: dyahis@apps.ipb.ac.id
}

pathway which also produces electochemical active compounds, it opens the chance to measure the degraded PAHs through oxidation process (Ghozali et al. 2020). The reduction step of extraction using organic solvent and diminishing the energy needed for analysis are two of the green analytical chemistry (GAC) principles. Accordingly, chemical analysis still needs to maintain its analytical performance (Tobiszewski et al. 2010). One of promising method for analysis is biosensor, because it matches the principles of GAC, like reducing organic solvent usage, miniaturization, less sample needed for analysis, and able to be applied for in situ analysis. Moreover it can be used to study the toxic effect of certain substances (Gałuszka et al. 2013). Considering these potentials of biosensor as a technology of monitoring environmental quality, this report focused on selecting bacteria isolates based on phenolic degradation, degradative enzymes, biofilm formation and their performance in sensing the target phenolic compounds.

\section{Materials and Methods}

\subsection{Materials and Instrumentation}

Screen printed carbon electrode (SPCE, Dropsense DRP 110), phosphate buffer solution 
pH 7.00 [ $\mathrm{NaCl} 154 \mathrm{mM}$, without $\mathrm{NaCl}]$, LuriaBertani (LB) and mineral media [solid and liquid], 1,2-dihidroksinaftalena (Merck), catechol (Merck), phenol (Merck), anthracene (Merck), naphthalene (Marck), sterile soil, GoTaq ${ }^{\circledR}$ Master Mix 2X, Bacterial isolates of Pseudomonas aeruginosa, Staphylococcus sciuri, and Bacillus amyloliquifaciens. Several equipments and instruments used here were: microplate reader, TaKaRa PCR Thermal Cycler, potensiostat/galvanostat E-DAQ.

\subsection{Rejuvenating the Isolates and Degradation of Organic Compounds}

The selected isolates were cultured on solid agar Luria-Bertani (LB) media. The selected isolates were cultured for \pm 18 hours in rotary shaker of 100 rpm, then harvested via centrifugation $(10,000$ rpm, 5 minute). The optical density (OD) value of the harvested isolates were around 1.00-1.50 (modification Iswantini et al. 2020).

Degradation of several aromatic compounds were conducted in two different ways: using bacterial culture in $200 \mu \mathrm{l}$ liquid LB on microplate 96 wells with naphthalene, anthracene, BTX (benzene, toluene, xylene), premium (octane $88,10 \mu \mathrm{L}$ ), and diesel oil $(10 \mu \mathrm{L})$ as tested compound. Second method used sterile soil enriched with mineral media (liquid M-9 100 gram/70 mL) with naphthalene and anthracene as tested compound ( $1 \mathrm{mg} / \mathrm{g}$ soil).

\subsection{Oxygenase Enzyme Gene Detection}

Polymerase chain reaction (PCR) was used to detect the oxygenase enzyme gene reported primers were used in this study, and some others were also built using the site on NCBI (www.ncbi. nlm.nih.gov) (Table 1). PCR method were conducted by using Master Mix 2.0 and TaKaRa Thermocycler.
PCR parameters for denaturation, annealing, and extension were $95^{\circ} \mathrm{C}, 50 / 58^{\circ} \mathrm{C}, 72^{\circ} \mathrm{C}$ respectively with 35 cycles.

\subsection{Biofilm Formation on SPCE}

Biofilm was formed by dropping $10 \mu \mathrm{L}$ bacterial suspension on screen printed carbon electrode (SPCE) working electrode. After the water had been evaporated on room temperature (assisted with wind blow), later the bacterial suspension was coated with $10 \mu \mathrm{L}$ agarose (1.00\%). Then the biofilm was incubated inside a sealed container on room temperature for 3 days before the electrochemical measurement.

\subsection{Electrochemical Measurement Settings}

SPCE (DRP C-110, Metrohm) with 3 electrodes system was used: (1) carbon electrode plate coated with biofilm as working electrode, (2) $\mathrm{Ag} / \mathrm{AgCl}$ as reference electrode, and (3) platinum electrode as auxiliary electrode. Measurement process was conducted using potentiostat/galvanostat E-DAQ v.2. Cyclic voltammetry was used as measurement mode type, with scan rate $100 \mathrm{mV} / \mathrm{s}$ and step 20 . There were 5 parameters which were evaluated for electrode analytical performance: precision, linearity, range, and limit of detection. Meanwhile the other electrochemical parameters of the biosensor were evaluated using Randles-Sevcik and Tafel were evaluated: exchange current density, diffusivity, reaction rate constant, transitional state sensitivity.

$$
\begin{gathered}
\mathrm{i}_{\mathrm{a}}=0.4463 \mathrm{nFAC} \sqrt{\frac{\mathrm{nFvD}}{\mathrm{RT}}} \text { (Randles-Sevcik equation) } \\
\ln \mathrm{i}_{\mathrm{a}}=\ln \mathrm{i}_{0}+\frac{(1-\alpha) \mathrm{nF}}{\mathrm{RT}} \eta \begin{array}{c}
\text { (cathodic current } \\
\text { Tafel equation) }
\end{array}
\end{gathered}
$$

Table 1. Primers which were used during experiment

\begin{tabular}{llll}
\hline \multirow{2}{*}{ Gene } & \multirow{2}{*}{ Species } & \multicolumn{1}{c}{ Forward } & Primer \\
\cline { 3 - 4 } & P. aeruginosa $^{(\mathrm{a})}$ & CACCTGATTCATGGCGATGAA & ACCATCAGATTGTGCGTCTGA \\
& S. sciuri(b) $^{(\mathrm{b})}$ & ATGGAATTCATGAAAAAAGGCGTAATGCGC & ATGCTCGAGTTAGGTCAGAACGGTCATGAA \\
& B. amyloliquifaciens $^{\text {Thy }}$ & TCAGGGAATGCGAAGTCAGG & TATGCCGTGCTGACAAAGGA \\
\hline CAT23 & P. aeruginosa ${ }^{(\mathrm{a})}$ & TGATCGAGATGGACCGTGACG & TCAGGTCAGCACGGTCATGAA \\
& S. sciuri & TCCAGCTGTTGTCCAAGTTGT & CCGCAAAGTTGGGTCATAA \\
& B. amyloliquifaciens & CTGTCACCGGCCTTTACCAT & CTTCATGATGCGGAAGCACG \\
\hline
\end{tabular}

NDO: naphthalene dioxygenase, CAT23: 2,3-catechol dioxygenase, (a) Marta et al. (2006), (b) Chang et al. (2011) 


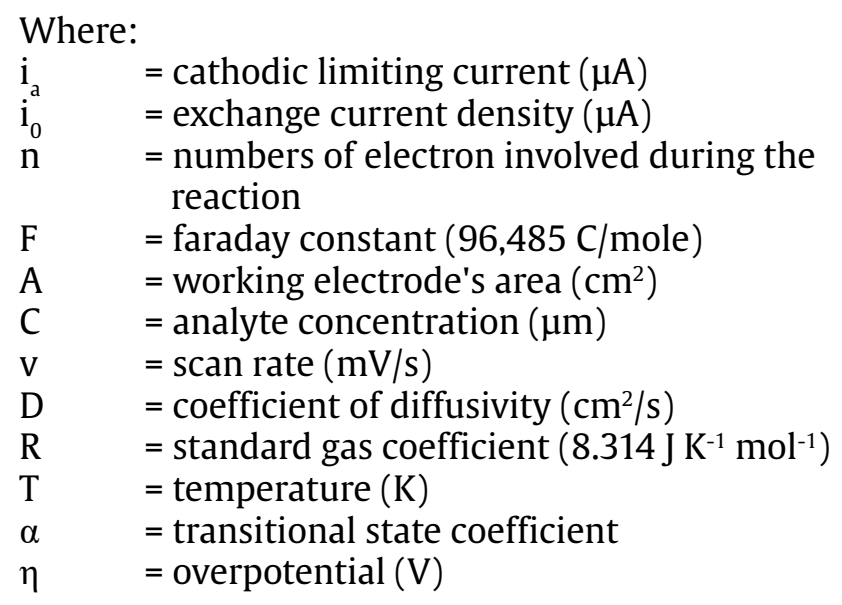

\section{Results}

\subsection{Degradation Capability of Isolates and Putative Gene}

There were two different approaches which were conducted for the research as stated in procedures. For the methods which using microplate 96 wells as the bacterial biofilm growth place, there were given two different condition: without $D$-glucose enrichment, and added with $D$-glucose $0.1 \%(\mathrm{w} / \mathrm{v})$. Both condition gave similar trend, yet adding glucose to the mineral liquid gave more higher tendency to grow the microbes (Figure 1). There were some different responses observed. B. amyloliquifaciens tended to grow slow, yet still giving an increasing optical density value up to the sixth day, meanwhile S. sciuri grew faster yet showed a decline since day fourth. $P$. aeruginosa showed little growth. Several negative responses were also observed, like $B$. amyloliquifaciens had no response on solar addition, meanwhile $P$. aeruginosa showed negative response on solar addition. Using single isolate on steril soil media had been conducted using naphthalene and anthracene (Figure 2). The degradation profile of each isolate showed similar result, with the average percentage of naphthalene and anthracene degradation between
75-84\% (naphthalene: $84.43 \pm 5.51 \%$; anthracene: $76.21 \pm 4.01 \%)$.

\subsection{Oxygenase Gene Detection}

The designed primer using NCBI databank were selected from putative gene data which were at the same genus (Staphylococcus sp. and Bacillus sp.). Some other reported primers were also tested in silico from all tested primers used through the PCR process, only two primers gave positive result: CAT23 for S. sciuri and $P$. aeruginosa (Figure 3 ). The PCR product of both isolate were detected on 358-391 bp and 1195-1209 bp. Primers characterizations are showed at Table 2. Table 3 showed the PCR product characteristics, especially the copy number gene. Since the copy number gene of CAT23 S. sciuri is higher, its PCR product was analysed further. By using the database NZ_CP022046.2:326039-326824 (NCBI database, S. sciuri strain FDAARGOS 286) against PCR product of CAT23 S. sciuri thrice replications, the alignment score against the gene source was ranging between $45-53 \%$ similarity only.

\subsection{Voltammogram Profile of Isolates}

All the measurement were conducted at $\mathrm{pH} 7.0$, since a lot of signal distortions happened with slightly more acidic or basic environment. More disrupting distortions were observed if the sample was more basic. It created more complex voltammogram profile. SPCE-Agarose was used as blank electrode, since agarose was used as supporting material for biofilm growth. Adding biofilm into the agarose showed a slight increase current produced (Figure $4 \mathrm{c}$ vs $4 \mathrm{~d}$ ). It affected the sensitivity value, showed by increasing gradient value of linear equation (Table 4 ). Yet, some significant value variations were noted through the measurement process (data not shown), even by using the same electrode at the second session measurement. The value of several other electochemical parameters, and voltammogram profile are shown in Table 5 and Figure 4. 

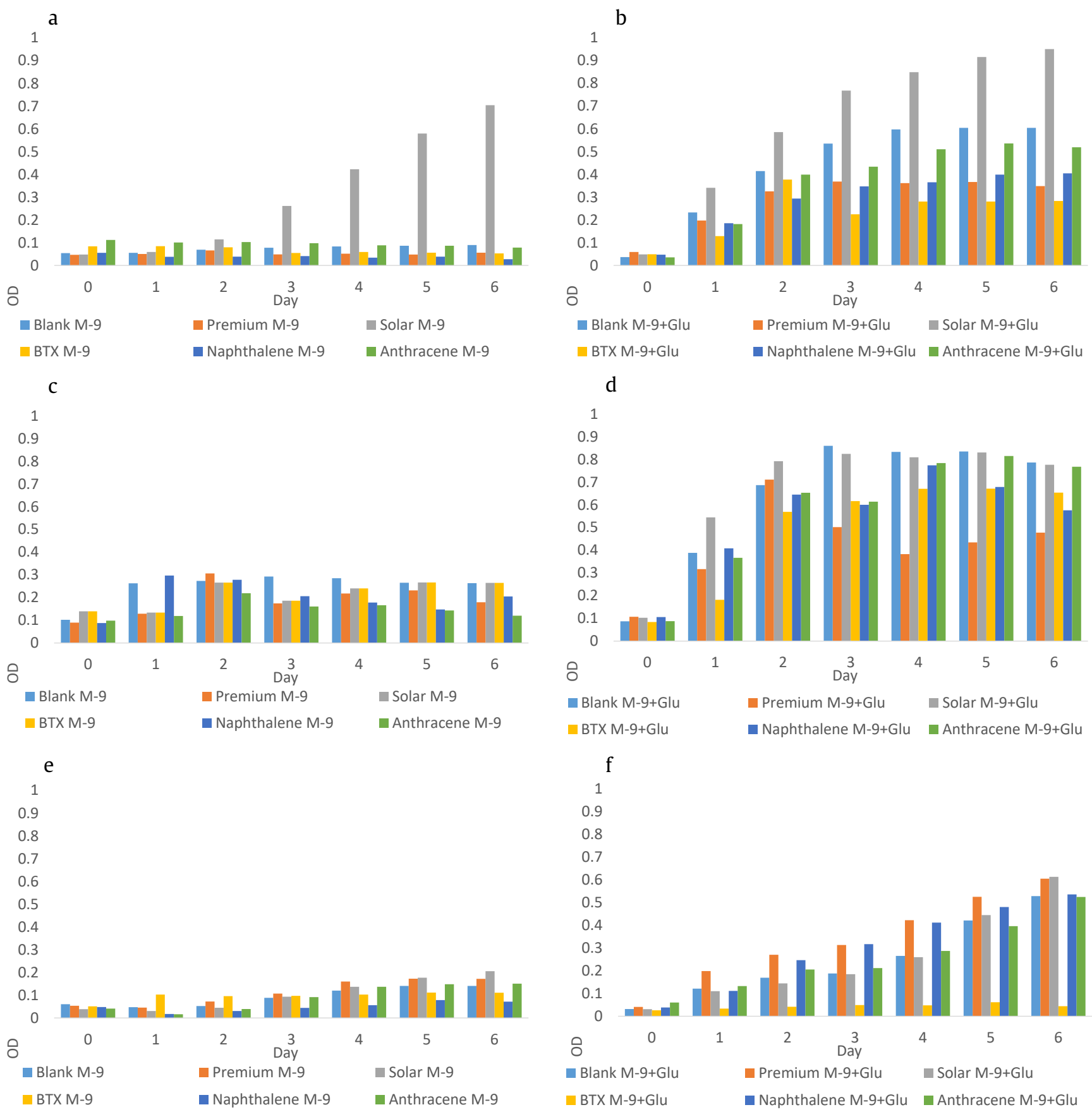

Figure 1. Growth colony profiles by optical density (OD) values: (a and b) P. aeruginosa, (c and d) S. sciuri, and (e and f) B. amyloliquifaciens. For (a), (c), and (e) were conducted without $D$-glucose addition, meanwhile (b), (d), and (f) with $0.1 \%$-glucose addition into liquid M-9 media. Notes, BTX: Benzene-Toluene-Xylene, Glu: 0.1\% D-glucose addition, M-9: minimum mineral media, Solar : Diesel Fuel ${ }^{\mathrm{TM}}$ Pertamina 


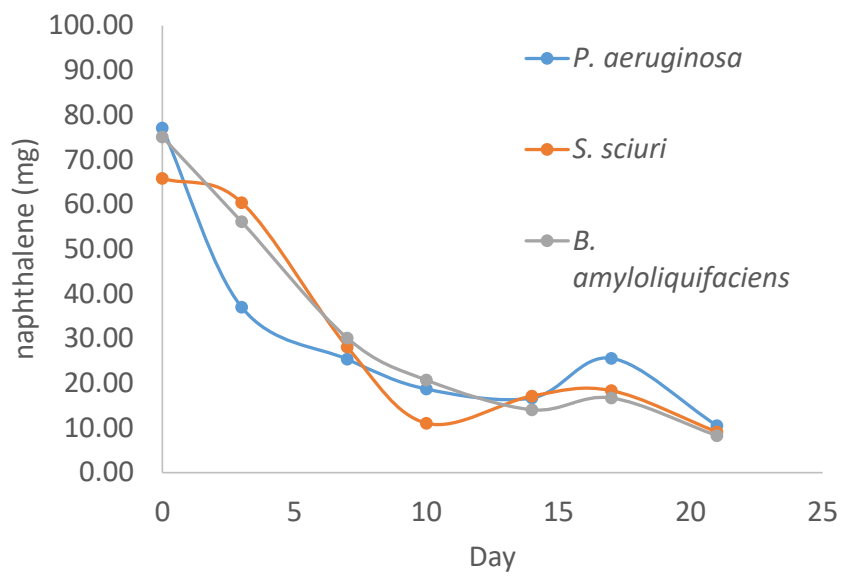

b

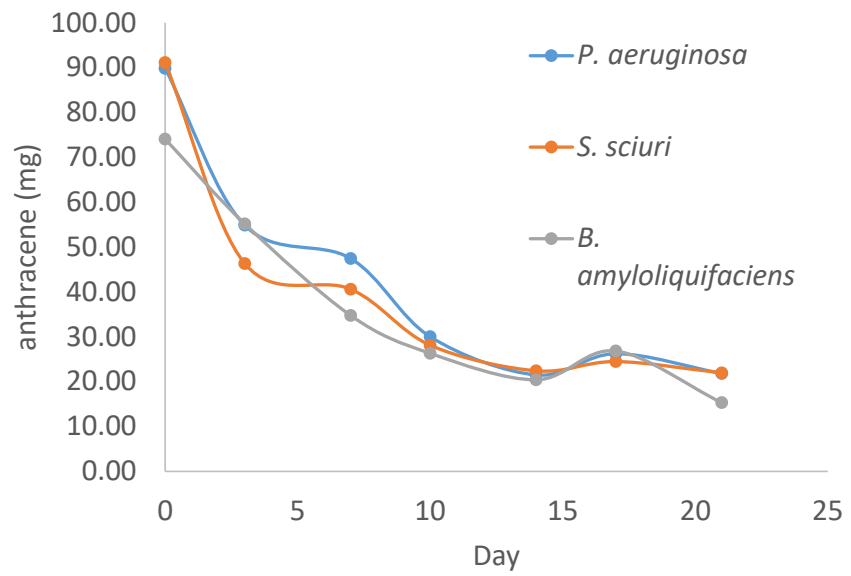

Figure 2. Degradation profile of aromatic compounds: (a) naphthalene, (b) anthracene

Table 2. Characterization of primers which were designed

\begin{tabular}{|c|c|c|c|c|c|c|c|c|}
\hline \multirow{2}{*}{ Gene } & \multirow{2}{*}{ Species } & \multicolumn{3}{|c|}{ Forward } & \multicolumn{3}{|c|}{ Reverse } & \multirow{2}{*}{ Product (bp) } \\
\hline & & $\% \mathrm{GC}$ & $\operatorname{Tm}\left({ }^{\circ} \mathrm{C}\right)$ & $\mathrm{n}$ & $\% \mathrm{GC}$ & $\operatorname{Tm}\left({ }^{\circ} \mathrm{C}\right)$ & $\mathrm{n}$ & \\
\hline \multirow[t]{3}{*}{$\mathrm{NDO}$} & P. aeruginosa & 48 & 59.4 & 21 & 48 & 59.4 & 21 & 2100 \\
\hline & S. sciuri & 40 & 68.0 & 30 & 47 & 70.4 & 30 & 900 \\
\hline & B. amyloliquifaciens & 55 & 60.5 & 20 & 50 & 58.5 & 20 & *495 \\
\hline \multirow[t]{3}{*}{ CAT23 } & P. aeruginosa & 57 & 63.5 & 21 & 52 & 61.3 & 21 & 650 \\
\hline & S. sciuri & 48 & 59.4 & 21 & 50 & 58.4 & 20 & $* 458$ \\
\hline & B. amyloliquifaciens & 55 & 60.5 & 20 & 55 & 60.5 & 20 & *537 \\
\hline
\end{tabular}

$\left.{ }^{*}\right)$ predicted PCR product result using NCBI

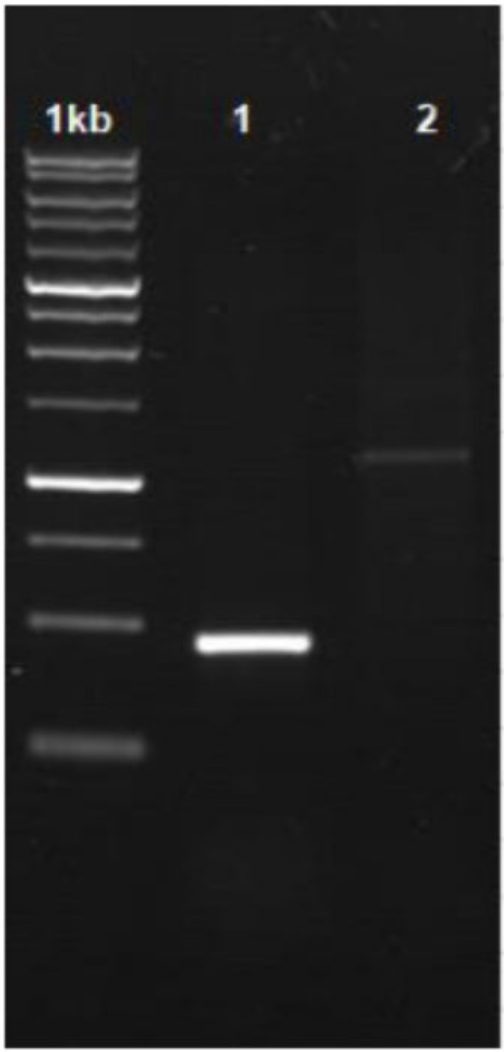

Figure 3. Electrophoresis of PCR products: 1 (S. sciuri CAT23), and 2. (P. aeruginosa CAT23)
Table 3. Analysis on intensity and copy number gene of PCR process

\begin{tabular}{lcccc}
\hline $\begin{array}{l}\text { Primer } \\
\text { isolate }\end{array}$ & $\begin{array}{c}\text { Intesity } \\
\text { (bit) }\end{array}$ & $\begin{array}{c}\text { Molecular } \\
\text { weight }(\mathrm{bp})\end{array}$ & $\begin{array}{l}{[\mathrm{DNA}]} \\
(\mu \mathrm{g} / \mu \mathrm{L})\end{array}$ & $\begin{array}{l}\text { Copy } \\
\text { number } \\
\text { gene }\end{array}$ \\
\hline $\begin{array}{l}\text { S. sciuri- } \\
\quad \text { CAT23 }\end{array}$ & 183906 & 358 & 0.356 & $1.82 \times 10^{12}$ \\
$\begin{array}{l}\text { P. aeruginosa- } \\
\text { CAT23 }\end{array}$ & 35391 & 1209 & 0.148 & $2.27 \times 10^{11}$ \\
\hline
\end{tabular}

\section{Discussion}

\subsection{Mechanism PAHs Degradation of Isolates and Putative Gene Oxygenase}

Based on the data taken during the degradation process, adding some simple sugar, like $D$-glucose, can increase the growth of microbes despite the presence of aromatic compounds. It is parallel with the same result reported by Reischke et al. (2015). Yet, it must be noted that some isolates showed negative response. It suggested that some isolates are more vulnerable for certain aromatic compounds or hydrophobic hydrocarbon substances. S. sciuri, showed to have tendency to degrade diesel oil, yet it is still inconclusive. By using sterile soil approach, the degradation rate can be easily measured. Yet, this approach is not suitable for naphthalene 
a

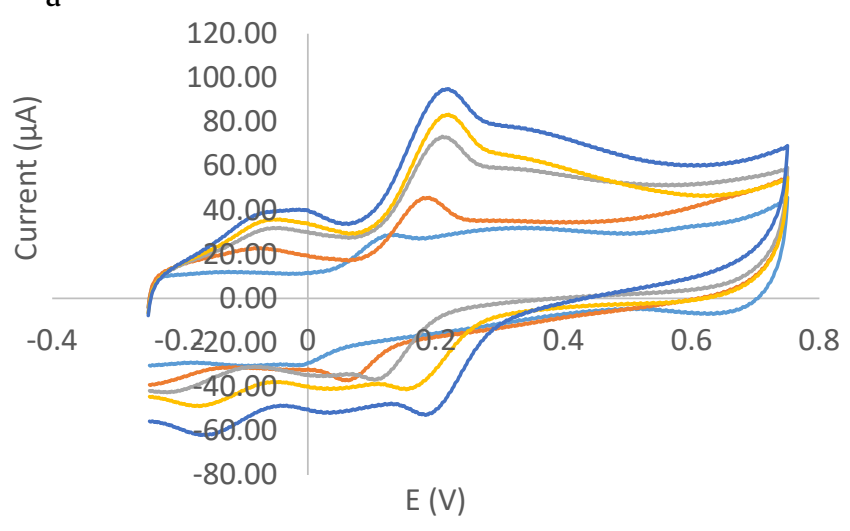

$-0.25-0.50-0.75-0.99-1.24$

C

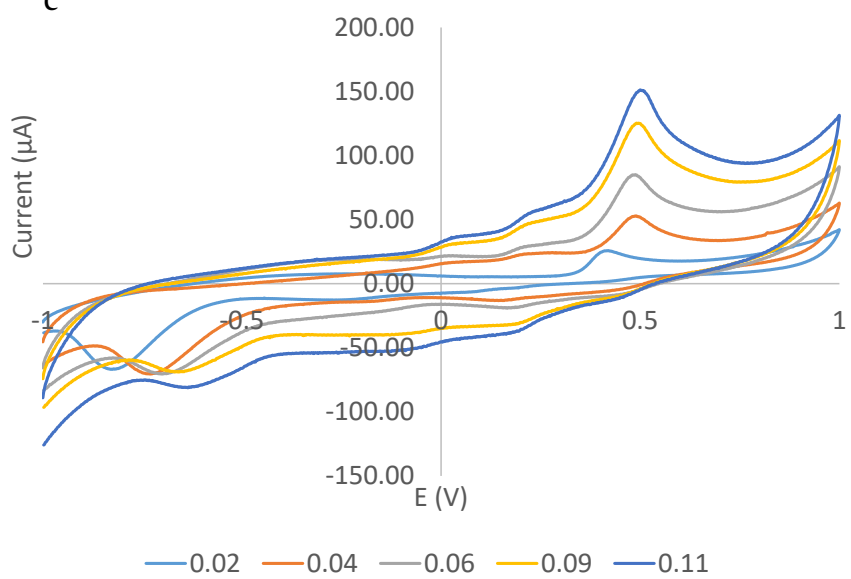

b

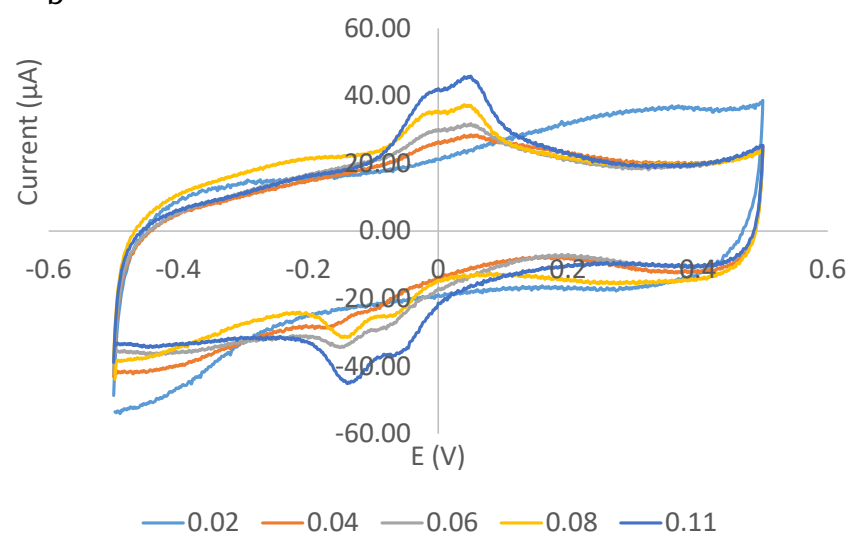

$\mathrm{d}$

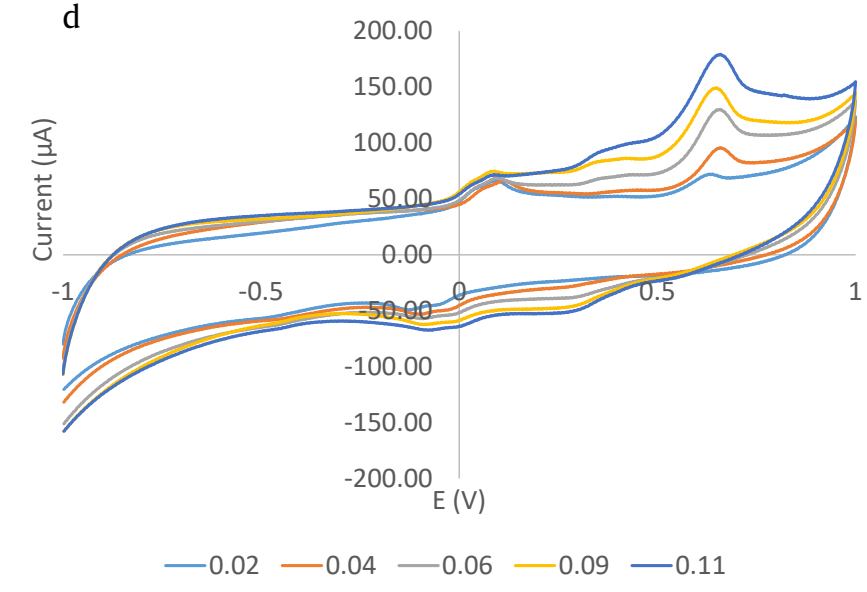

Figure 4. Voltammogram profile of biofilm P. aeruginosa: (a) catechol,(b) 1,2-dihydroxynaphthalene, (c) phenol; meanwhile (d) is voltammogram profile of phenol measured on SPCE-Agarose

Table 4. Analytical performance of each biofilm for molecule samples measurement

\begin{tabular}{llcccc}
\hline \multirow{2}{*}{ Sample } & \multirow{2}{*}{ Isolate } & \multicolumn{3}{c}{ Parameters } \\
\cline { 3 - 6 } Catechol & SPCE+Agarose & Intercept $(\mu \mathrm{A})$ & Gradient $(\mu \mathrm{A} / \mathrm{mM})$ & \multicolumn{1}{c}{$\mathrm{R}^{2}$} & $\mathrm{LOD}^{*}(\mu \mathrm{M})$ \\
\hline \multirow{4}{*}{ 1,2-dihydroxynaphthalene } & P.aeruginosa & 3.30 & 28.10 & $98.58 \%$ & 180 \\
& S. sciuri & 3.75 & 47.87 & $96.06 \%$ & 300 \\
& B. amyloliquifaciens & 4.78 & 39.34 & $98.14 \%$ & 210 \\
& SPCE+Agarose & -0.26 & 51.02 & $98.66 \%$ & 170 \\
\hline \multirow{4}{*}{ Phenol } & P.aeruginosa & -4.84 & 189.02 & $97.18 \%$ & 20 \\
& S. sciuri & -6.65 & 259.59 & $94.09 \%$ & 30 \\
& B. amyloliquifaciens & -7.57 & 308.24 & $93.24 \%$ & 30 \\
& SPCE+Agarose & 7.62 & 369.20 & $96.57 \%$ & 20 \\
\hline & P.aeruginosa & 30.13 & 17.71 & 0.97 & 0.32 \\
& S. sciuri & 13.63 & 36.81 & 0.88 & 0.63 \\
& B. amyloliquifaciens & 13.63 & 40.95 & 0.93 & 0.46 \\
& & 46.19 & 0.96 & 0.34
\end{tabular}

${ }^{*}$ ) Potential LOD value given from single electrode measurement 
Table 5. Several other parameters for measurement molecule samples measurement

\begin{tabular}{|c|c|c|c|c|c|}
\hline \multirow{2}{*}{ Sample } & \multirow{2}{*}{ Isolate } & \multicolumn{4}{|c|}{ Parameters } \\
\hline & & Diffusivity* & $\begin{array}{l}\text { Exchange current } \\
\text { density }\left(\mathrm{A} \mathrm{cm}^{-2}\right)\end{array}$ & $\begin{array}{l}\text { Transitional } \\
\text { state constant }\end{array}$ & $\begin{array}{l}\text { Reaction } \\
\text { rate }(\mathrm{M} / \mathrm{s})\end{array}$ \\
\hline \multirow{4}{*}{ Catechol } & SPCE+Agarose & $3.35 \times 10^{-7}$ & $5.54 \times 10^{-6}$ & 0.81 & $4.61 \times 10^{-9}$ \\
\hline & P.aeruginosa & $1.62 \times 10^{-6}$ & $1.95 \times 10^{-5}$ & 0.87 & $1.62 \times 10^{-7}$ \\
\hline & S. sciuri & $9.51 \times 10^{-7}$ & $1.91 \times 10^{-5}$ & 0.88 & $1.59 \times 10^{-7}$ \\
\hline & B. amyloliquifaciens & $7.71 \times 10^{-7}$ & $1.80 \times 10^{-5}$ & 0.88 & $1.50 \times 10^{-7}$ \\
\hline \multirow{4}{*}{ 1,2-dihydroxynaphthalene } & SPCE+Agarose & $3.32 \times 10^{-7}$ & $4.35 \times 10^{-5}$ & 0.85 & $4.27 \times 10^{-6}$ \\
\hline & P.aeruginosa & $2.67 \times 10^{-7}$ & $3.59 \times 10^{-5}$ & 0.90 & $3.52 \times 10^{-6}$ \\
\hline & S. sciuri & $2.60 \times 10^{-7}$ & $2.34 \times 10^{-5}$ & 0.89 & $2.30 \times 10^{-6}$ \\
\hline & B. amyloliquifaciens & $6.36 \times 10^{-7}$ & $3.45 \times 10^{-5}$ & 0.87 & $3.38 \times 10^{-6}$ \\
\hline \multirow{4}{*}{ Phenol } & SPCE+Agarose & $2.04 \times 10^{-7}$ & $6.95 \times 10^{-6}$ & 0.84 & $1.16 \times 10^{-7}$ \\
\hline & P.aeruginosa & $2.68 \times 10^{-5}$ & $1.31 \times 10^{-5}$ & 0.90 & $2.18 \times 10^{-7}$ \\
\hline & S. sciuri & $1.09 \times 10^{-6}$ & $1.39 \times 10^{-5}$ & 0.89 & $2.31 \times 10^{-7}$ \\
\hline & B. amyloliquifaciens & $1.39 \times 10^{-6}$ & $1.61 \times 10^{-5}$ & 0.90 & $2.67 \times 10^{-7}$ \\
\hline
\end{tabular}

*) Diffusivity constant were taken when the concentration was $1.25 \mathrm{mM}$

and anthracene due to the state of the matters. Both compounds are on solid state, thus the homogenousity is somewhat questionable. Besides, the sample was very wet, muddy-like, complicating the sampling design. Using only single sample point data, it was shown that both substance, anthracene and naphthalene, can be degraded by single isolate culture with the similar degradation profile. Both degradation profile are not so significantly different due to the structure difference is not so dissimilar (Fulekar 2017).

According to primer criteria (Table 2), some primers had G-C percentage which were less than $50 \%$. All primers were designed between 20-21 bp, which is conveniently used. Except, primer for NDO S. sciuri which was reported by Chang (2011) was designed based on the highly conserved area of CAT23. After PCR process primer for NDO putative gene reported from Chang et al. (2011) showed no result. Yet it was reported to appear at $900 \mathrm{bp}$. The PCR products had no any result, except the amplification of CAT23 from $S$. sciuri and $P$. aeruginosa. The low quality of the PCR products may be the result of several contributing factors, either from DNA extraction, mixing PCR reagent, un-optimized PCR parameter condition, even during sequencing process (Febria et al. 2011).

\subsection{Analytical Performance of Biosensor}

Some relatively big variations were noted between the electrodes with the same biofilm microbe. Even with the same electrode, the measurement result changed drastically. It indicates the fabrication repeatability is still low (data not shown). Moreover, it also indicates the biofilm only performs well for single measurement data taken, even with the same exact concentration. The homogenity of the bacteria condition and also the immobilization process are pointed out as the most influencing factor. One of the parameters of analytical performance is limit of detection (LOD) and limit of quantization (LOQ). Both parameters showed no significant difference between blank electrode with biofilm coated electrode, even it produced higher LOD and LOQ value. On the other hand, the sensitivity value of blank electrode tended to be lower than biofilm coated electrode (Table 4). Figure 4 shows that the voltammogram profiles of biofilm-coated electrodes have thicker baseline current. It indicates a higher conductive current produced during the measurement. This contributes to the higher value of LOD and LOQ. High LOD and LOQ value are not prefered for constructing a new measurement method, yet its higher sensitivity indicates the biofilm can activates the catalytic current. Current density state, transitional state coefficient, diffution coefficient, and coefficient rate showed similar pattern between all biofilm and blank electrode (Table 5). Catechol and phenol tended to be more attracted to the electrode based on diffusivity constant, and when it went to electrochemical reaction with the electrode, it favors the product state, indicated by $\alpha$ values which are almost 1 . Yet, their coefficient rate and current density showed a common lower value for the biofilm-coated electrode. This suggests the thickness of the biofilm supported with agarose need to be optimized, yet the reaction rate indicated by the coefficient rate is somewhat similar. Ghozali et al. (2020) reported the usage of PQQ-GDH (glucose dehydrogenase) as the bioreceptor for detecting 1,2-dihydroxynaphthalene and catechol. The proposed mechanism of detection is shown at Figure 5. 


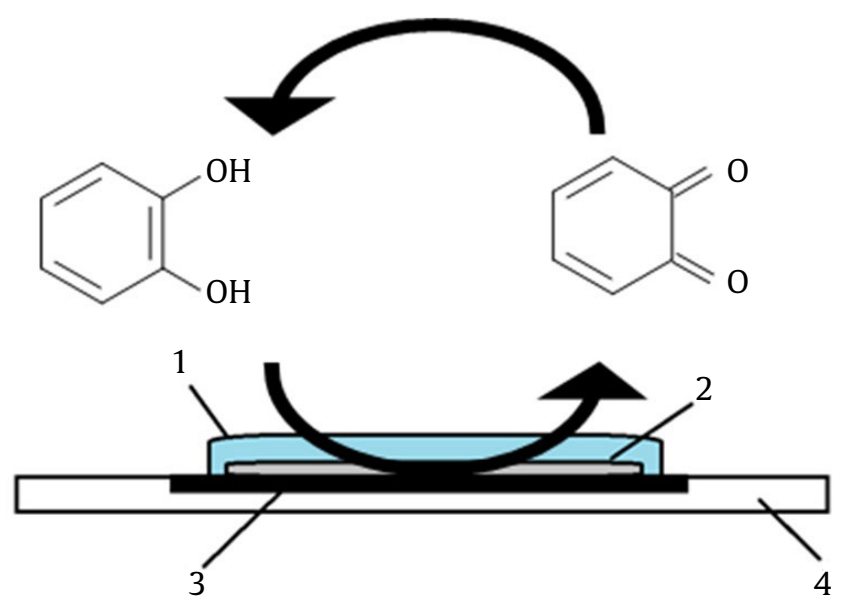

Figure 5. Reaction mechanism and cross-section of SPCEbiofilm with agarose as supporting material: (1) agarose membrane $(1.0 \%, 10 \mu \mathrm{L})$, (2) bacterial biofilm $10 \mu \mathrm{L}$, (3) working electrode of SPCE, (4) SPCE plate

\subsection{General Discussion}

Phenolic compounds, like phenol, catechol, resorcinol, and naphthalenadiol, are potential pollutants in the aquatic environment. The presence of phenolic compounds is also an indication of the degradation of organic compounds, such as PAHs, or the presence of organic waste (Anku et al. 2017). Therefore, many phenolic compounds were the target of the pollutant compounds being analyzed. Based on the existing transduction system, phenolic compounds are more likely to be analyzed using an electrochemical approach in biosensor applications. Because phenolic compounds intrinsically can perform redox reactions and are easier than the operating system for the device (Fakhruddin and Karim 2012).

Comparing the measurement with fluorimeter tandem HPLC (Marrubini et al. 2005), LOD value and sensitivity of conventional method techniques are almost commensurate with the analytic performance of biosensors, especially enzymatic biosensors. Bacteria as bioreceptors also have the potential to improve their analytical performance, with the addition of polymers that are electroactive (such as Nafion) or carry out genetic engineering (Zhang et al. 2018). Judging from the LOD value, if the phenol threshold is taken as the minimum standard of concentration, which is considered safe according to KEP No. 51/MENLH/10/1995, all types of biosensors that have been reported are capable to detect (0.5$1.0 \mathrm{mg} / \mathrm{L}$ ). Likewise, if the phenol concentration threshold reference is used for drinking water, 0.002
mg/L (Hudori and Yulianto 2011). Thus, in terms of analytical performance, biosensor has the potential as a complementary method to conventional measurement techniques.

There are 12 basic principles of the GAC that have been offered (Gałuszka et al. 2013). This biosensor technique according to the GAC principles includes the principles of waste minimization, environmentally friendly reagents, and energy efficiency; four of the five core principles of the GAC. However, there are at least two certain aspects that need attention, especially in the application process of biosensor techniques. First, from the many journal reports that have been published, all of them prioritize maximum analytical performance. It is characterized by a high sensitivity indicator and a low LOD value. However, at the same time, the use of several chemical materials has the potential to increase operational costs per one unit of analysis, such as gold nanoparticles. The cost factor of this analysis has not been included as an important factor in the aspect of GAC.

Second, disregarding the internal factors of the tool's fabrication, conventional methods, such as HPLC and GC-MS, already have standards in determining column types and homogeneously been fabricated. Because it uses an inorganic type of material, the conformity of the material is more homogenous and easier to replicate. Yet, the uniformity of biosensor is an important key factor in the development of the method, either by intrinsic properties or when applying it as a biofilm. In the case of bacteria as bioreceptor, further validation is needed regarding the identity and standardization of their physiological processes. Compared with enzymes, the use of bacteria can reduce the cost of making fabrications, especially the genetically modified bacteria.

\section{Conclusion}

The bacteria that have been characterized previously were observed potentially as biodegraders of aromatic compounds. This has been observed physiologically from the decreasing of the PAHs amount and the oxygenase gene detection. Regarding the function of bacterial isolates as biodegradators, it is necessary to conduct more experiment of the degradation effectiveness, degradation rate, and increasing the bacteria performance of degradation, both in batch and landfarming scale. With the same bacterial isolate, its usage can be further explored for biofuel cell technology, or detection of phenolic compounds. Meanwhile, for the analytical 
performance aspect of the biosensor, further research is needed on the biofilm viability optimization, validation, interference of other molecules and ions, and application of measurement in situ, according to the GAC principles.

\section{Acknowledgements}

Authors give thank and gratitude to Ministry of Research and Technology of the Republic of Indonesia (Kemenristek-BRIN) for supporting this research through grant scheme No. 1/E1/KP.PTNBH/2021 and No. 2093/IT3.L1/PN/2021.

\section{References}

Anku WW et al. 2017. Phenolic Compounds in Water: Sources, Reactivity, Toxicity and Treatment Methods. In: Marcos Soto-Hernandez, Mariana Palma-Tenango and Maria del Rosario Garcia-Mateos (Eds.). Phenolic CompoundsNatural Sources, Importance and Applications. London: IntechOpen. pp. 420-443.

Chang $\mathrm{CH}$ et al. 2011. Staphylococcus sp. KW-07 contains nahH gene encoding catechol 2,3-dioxygenase for phenanthrene degradation and a test in soil microcosm. Int Biorem Biodeg 65:198-203. DOI:10.1016/j. ibiod.2010.11.003

Fakhruddin ANM, Karim F. 2012. Recent advances in the development of biosensor for phenol: a review. Rev Enviro Sci Biotech 11:261-274. DOI:10.1007/s11157012-9268-9

Febria FA et al. 2011. Identifikasi gen penyandi piren dioksigenase pada isolate bakteri pendegradasi piren. Berita Biol 10:781-786.

Fernandez-Valera $\mathrm{R}$ et al. 2010. Selecting a reduced suite of diagnostic ratios calculated between petroleum biomarkers and polycyclic aromatic hydrocarbons to characterize a set of crude oils. J Chrom A 1217:82798289.

Fulekar MH. 2017. Microbial degradation of petrochemical waste-polycyclic aromatic hydrocarbons. Bioresour Bioprocess 4:28. DOI:10.1186/s40643-017-0158-4

Gałuszka A et al. 2013. The 12 principles of green analytical chemistry and the SIGNIFICANCE mnemonic of green analytical practices. Trends in Anal Chem 50:78-84 DOI:10.1016/j.trac.2013.04.010
Ghozali Ali A et al. 2020. Sensitive simultaneous determination 1,2-dihydroxynaphthalene and catechol by amperometric biosensor. Analytical Science 37:991995. DOI:10.2116/analsci.20P393

Hudori, Yulianto A. 2011. Penurunan fenol melalui proses. J Sains Tek Ling 3:66-72.

Iswantini D et al. 2020. Activity and stability of the alcohol biosensor using acetobacter aceti biofilm on screenprinted carbon electrode. Hayati Journal of Bioscience 27:24-30 DOI:10.4308/hib.27.1.24

Liu FF et al. 2014. Mixture effects of benzene, toluene, ethylbenzene, and xylenes (BTEX) on lung carcinoma cells via a hanging drop air exposure system. Chemical Research in Toxicology 27:952-959 DOI:10.1021/ tx5000552

Marrubini G et al. 2005. Direct analysis of phenol, catechol and hydroquinone in human urine by coupled-column HPLC with fluorimetric detection. Chromatographia 62:25-31. DOI:10.1365/s10337-005-0570-3

Marta I et al. 2006. Contaminated sites: assessment of the metabolism growth and genetic characterization of wild-type microbial strains able to degrade naphthalene. Prev Today 2:35-50.

Minarghi FS et al. 2019. Monitoring of polycyclic aromatic hydrocarbon contamination at four oil spill sites using fluorescence spectroscopy coupled with parallel factorprincipal component analysis. Env Sci: Proc Imp 21:413426. DOI:10.1039/c8em00493e

Pampanin DM, Sydnes MAU. 2012. Polycylic aromatic hydrocarbons a constituent of petroleum: presence and influence in aquatic environment. Hydrocarbons, Chapter 5:82-118 DOI:10.5772/48176

Reischke S et al. 2015. Threshold concentration of glucose for bacterial growth in soil. Soil Biol and Biochem 80:218-223. DOI:10.1016/j.soilbio.2014.10.012

Tobiszewski M et al. 2010. Green analytical chemistry-theory and practice.Chem SocRev 39:2869-2878 DOI: 10.1039/ B926439F

Yin $\mathrm{F}$ et al. 2015. Long-term monitoring data to describe the fate of polycyclic aromatic hydrocarbons in deepwater horizon oil submerged off alabama's beaches. Sci Tot envir 508:46-56.

Zhang Z et al. 2018. Detection of catechol using an electrochemical biosensor based on engineered Escherichia coli cells that surface-display laccase. Anal Chim Act 1009:65-72. DOI:10.1016/j.aca.2018.01.008 